\title{
Ensayo \\ La política cultural en el siglo XXI: entre la diversidad y el entorno digital
}

Fecha de recepción : 2020-07-08 • Fecha de aceptación:2020-08-17 • Fecha de publicación:2020-09-10

Belén Fiallos Quinteros

Universidad de Artes de La Habana (ISA), Cuba

belen.fiallos@gmail.com

https://orcid.org/0000-0003-3136-4643

\section{Resumen}

En este ensayo se abordan las políticas culturales en el siglo XXI, específicamente las relacionadas con el entorno digital. Se parte de un breve análisis de la Convención sobre la Protección y la Promoción de la Diversidad de las Expresiones Culturales (UNESCO, 2005). En segundo lugar, se abordan categorías y algunas problemáticas que surgen a partir de las nuevas dinámicas que emergen del mundo contemporáneo producto de la globalización, Internet, tecnologías y los nuevos procesos sociales en relación a la diversidad cultural y el desarrollo de la industria cultural. Para concluir, se apunta a conceptualizar cuáles son las políticas y estrategias para la protección y promoción de la diversidad de las industrias culturales en el entorno digital.

Palabras clave: UNESCO, globalización, diversidad cultural, industrias culturales, políticas culturales, digitalización.

\footnotetext{
Abstract

This essay addresses cultural policies in the 21 st century, specifically those related to the digital environment. It starts from a brief analysis of the Convention on the Protection and Promotion of the
} 
Diversity of Cultural Expressions (UNESCO, 2005). Secondly, categories and some problems that arise from the new dynamics that emerge from the contemporary world as a result of globalization, the internet, technologies and new social processes in relation to cultural diversity and the development of cultural industry are addressed. To conclude, it aims to conceptualize what are the policies and strategies for the protection and promotion of the diversity of cultural industries in the digital environment.

Keywords: UNESCO, globalization, cultural diversity, cultural industries, cultural policies, digitalization. 


\section{Introducción}

Las sociedades actuales se caracterizan por ser complejas, globalizadas, diversas, interculturales e interconectadas. Ellas enfrentan nuevos desafíos debido, entre otros factores, al entorno digital, que es el espacio real y virtual en el cual interactúan las herramientas que existen en Internet y están conformadas por las redes sociales, blogs, email, tiendas en línea, intranet, diferentes plataformas ${ }^{1}$ y aplicaciones, y las nuevas tecnologías digitales que comprenden equipos de producción, el Internet de banda ancha, la televisión on demand, teléfonos inteligentes, los QR, cámaras digitales, dispositivos personalizados de consumo, entre otras. Todos estos factores relacionados con la cultura han generado un importante debate que ha comenzado a cobrar fuerza en varios ámbitos y que se ha centrado en la problemática de cómo proteger y promover a través de políticas públicas la diversidad cultural de los bienes y expresiones culturales de las diferentes sociedades.

El entorno digital ofrece nuevos lenguajes y la posibilidad de un mundo sin fronteras, crea nuevas relaciones y espacios de comunicación y, por otra parte, influye en las industrias culturales y medios de comunicación, produciendo cambios en los procesos de producción artística y la creatividad.

Por otro lado, los procesos de globalización, bien conocidos en el campo económico, se han extendido también a los sectores ambientales, tecnológicos y culturales. Precisamente, uno de los debates más trascendentales se direcciona a este último campo, interesándose por reflexionar sobre cuáles son las políticas públicas y a través de qué acciones se puede proteger y promover la diversidad de los bienes y servicios culturales. En este sentido, las industrias reclaman especial atención ya que estas han sido uno de los sectores más impactados por el desarrollo de las nuevas tecnologías y el entorno digital.

En un mundo globalizado, donde la cultura se convierte, en la mayoría de los casos, en mercancía y tiende a la homogeneización, los factores del entorno digital ofrecen oportunidades y amenazas para la diversidad, su defensa "no implica necesariamente una única y determinada intervención social o de política cultural. Su nivel de ambivalencia puede llevar a fenómenos de reconocimiento y promoción de lo diferente o minoritario o, al contrario, a la asimilación artificial de las diferencias, cuando no a lo segregación de colectivos" Zallo (2017, pp. 96-97). Por lo tanto, las políticas culturales bajo este contexto deben formularse e implementarse en relación a las nuevas dinámicas que se producen en la actualidad, para asegurar la creación, distribución y un acceso equitativo y diverso de los contenidos culturales.

En el debate sobre las políticas culturales para la protección y promoción de la diversidad cultural, el interés ha girado en torno a conocer cuáles son los mecanismos para visibilizar los diferentes grupos que conforman una sociedad, determinar cuáles son sus expresiones culturales y su manera de relacionarse, cómo se distribuyen y quién las consume. En ese sentido, La

1 Google, Amazon, Facebook y Apple se han posicionado, junto a Spotify, Netflix, Itunes, entre otros, de una manera dominante en el entorno digital, creando un importante poder mediático y cultural. Su presencia ha impactado de manera significativa en el campo de la industria cultural. 
Organización de las Naciones Unidas para la Educación, la Ciencia y la Cultura (UNESCO) ha contribuido con importantes reflexiones y documentos internacionales aportando una serie de lineamientos de política cultural, así como una propuesta de indicadores para la formulación de la misma.

\section{La Convención de 2005: un instrumento regulador de las expresiones y bienes culturales en el contexto global}

En la actualidad, los procesos de globalización aceleran el flujo de la información, la libre circulación de personas, mensajes, intercambios culturales y económicos. Todos estos procesos imponen una serie de normas y maneras de relacionarse que condicionan los sistemas existentes en varios campos, particularmente en el de estudio.

La transformación estructural de los procesos en el campo cultural obliga a replantearse su concepto mismo y sus modelos de análisis. Hoy ya no se puede pensar en esta noción como un espacio de la alta cultura y las bellas artes. Vich (2014) enfatiza en la conceptualización de la cultura como una vertiente antropológica, este la ubica como un dispositivo socializador que regula las relaciones sociales, produce realidad y al mismo tiempo es el soporte de esta realidad. Es decir, la cultura entendida como un factor protagónico para la construcción de procesos y propiciadora de relaciones interculturales. Por otro lado, Yúdice (2002, p. 43) afirma que "el recurso de la cultura sustenta la performatividad en cuanto lógica fundamental de la vida social hoy".

En efecto, la cultura ha dejado de ser un concepto estático para transformarse en un objeto de reflexión e interpretación; ha perdido su carácter patrimonial para convertirse en un dinamizador de procesos -extendiéndose hasta el campo digital- y en una herramienta de transformación social. La cultura, hoy en día, es un importante pilar para el desarrollo económico, social y humano (Martinell, 2010), además de constituirse en una herramienta fundamental para la gestión de la diversidad como parte esencial de la cultura.

La reflexión académica actual asocia la diversidad cultural con categorías como: la identidad, la protección de los grupos minoritarios y con la idea del otro. Ochoa (2008) sostiene que la diversidad cultural puede ser un instrumento para enfatizar la heterogeneidad y Georges Yúdice nos recuerda que "la diversidad no tiene que ver solo con los contenidos, por importantes que estos sean. Tiene que ver también con las plataformas en las que se determina la velocidad y las condiciones de circulación de los contenidos" (2017, p.124). Sin embargo, a pesar de las múltiples aproximaciones al término, no se ha llegado a un consenso para su definición.

En el mundo contemporáneo los procesos de globalización asociados a las lógicas del mercado y a las tecnologías digitales han desdibujado las fronteras simbólicas. Las distintas culturas e identidades circulan en escenarios reales y virtuales redefiniéndose constantemente. Hoy, estos escenarios reclaman el reconocimiento y respeto por las diferentes diversidades, ya sean étnicas, cultura o las categorías identitarias que se han reconocido en los últimos años: género, edad, profesiones, nickname, etc (Appadurai, 2014, pp.60-61).

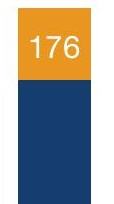


De ahí, que el reconocimiento de estas diversidades necesite de medidas y estrategias con enfoques amplios destinados a las minorías y a los grupos discriminados para lograr un ambiente incluyente que ayude a visualizar las identidades invisibilizadas, defender sus derechos individuales y colectivos para, de esta manera, oxigenar los cauces democráticos.

En el contexto global, la lucha por la legitimidad de la democracia y la defensa de la diversidad de las expresiones culturales se ha convertido en uno de los mayores retos en la actualidad. Martín Barbero (2008) propone una negociación de la diversidad con la globalización, basada en el conocimiento de las historias de los pueblos, es decir, fundamentada en las experiencias y memorias emanadas de la diversidad cultural, puesto que "para ser reconocidos necesitamos contar nuestro relato, pues no existe identidad sin narración, ya que esta no es solo expresiva, sino constitutiva de lo que somos".

Así mismo, la diversidad cultural reclama una organización de estas expresiones y la gestión de las diferencias, a través de acciones orientadas a protegerla y fomentarla. Exige también nuevas instituciones más democráticas, capaces de solucionar los conflictos que emergen de los nuevos contextos, tales como los intercambios culturales y de mercado, los diferentes modos de acceso y consumo cultural, la concentración empresarial y la homogeneización de los contenidos culturales.

La noción de diversidad cultural, y posteriormente el debate sobre la promoción y protección de los bienes y expresiones culturales, ha sido un tema prioritario para la UNESCO. Este interés se ve reflejado en varios documentos y declaraciones ${ }^{2}$, fundamentalmente en la Declaración universal sobre diversidad cultural (2001) que reconoce la cultura como un factor clave para el desarrollo y a la diversidad cultural como medio para garantizar la paz y la democracia en las sociedades y en la Convención para la Protección y Promoción de las Expresiones y Bienes Culturales que define a la diversidad como las distintas formas en que se expresan y transmiten los diferentes grupos que conforman las sociedades, así como los "distintos modos de creación artística, producción, difusión, distribución y disfrute de las expresiones culturales, cualesquiera que sean los medios y tecnologías utilizados" (UNESCO, 2005).

Precisamente, en el año de 2005, la UNESCO adopta los términos de la Convención sobre la Protección y la Promoción de la Diversidad de las Expresiones Culturales, celebrada en el marco de la $33^{a}$ Reunión de la Conferencia General de las Naciones Unidas celebrada en París. Este documento es un instrumento internacional vinculante que reconoce el carácter de los bienes, servicios y actividades culturales, más allá de su valor comercial, como transmisores de identidad, valores y sentidos. Es uno de los instrumentos de mayor importancia para el reconocimiento de la diversidad cultural como fuente de creatividad, innovación e inclusión social.

2 Colección sobre la unidad y diversidad cultural (1953); Nuestra diversidad creativa (1996); Reporte mundial sobre cultura, creatividad y mercados (2000); Reporte mundial sobre la cultura. Diversidad cultural, conflicto y pluralismo (2000); Declaración universal sobre diversidad cultural (2001) Informe sobre el desarrollo humano. La libertad cultural en el mundo diverso de hoy (2004); Convención para la protección y promoción de las expresiones y bienes culturales (2005); Informe Mundial de la Unesco. Invertir en la diversidad cultural y el diálogo intercultural (2010). 
Por otro lado, establece un marco para la gestión y la elaboración de políticas culturales para la diversidad de las expresiones culturales y es un mecanismo para reforzar la cooperación cultural internacional y regional. Relaciona a la diversidad con el debate de la cultura y el comercio. Además, la vincula con procesos de desarrollo y con la expresión creadora de los bienes y servicios culturales. En ella se solicita una nueva comprensión de la relación entre diversidad, diálogo y desarrollo para impulsar marcos de acción y lineamientos de política cultural.

Su importancia radica en ser el primer instrumento internacional que reconoce a los bienes culturales como vectores de identidad, trascendiendo el carácter comercial que se les había atribuido hasta ese momento. Este debate había sido abordado exclusivamente en los tratados de libre comercio, específicamente en la Ronda de negociaciones del Doha como parte de la Organización Mundial de Comercio (OMC), quienes promulgaban el régimen jurídico de la excepción cultural, es decir que permitía aplicar excepciones de cuotas y subvenciones a los productos culturales.

No hay duda que la Convención (2005) de la UNESCO es un aporte relevante para el derecho internacional, por cuanto plantea un nuevo marco para la gestión y la elaboración de políticas culturales enfocadas en la protección y promoción la diversidad de las expresiones culturales frente al peligro de la homogenización y un nuevo sistema de gobernanza para la cultura a través de la colaboración del Estado, el sector público y privado, la sociedad civil y la cooperación internacional. Sin embargo, a más de diez años de la aprobación de la Convención, no se pueden encontrar ejemplos paradigmáticos sobre su implementación, ni resultados del impacto ${ }^{3}$ a nivel internacional (Albornoz y Leiva, 2017a).

Ante este escenario, el desafío consiste en implementar eficazmente los lineamientos de política orientados en la Convención ya que, por más avanzados que sean conceptualmente, corren el peligro de quedar en simple retórica, con lo cual, y como consecuencia, las expresiones y bienes culturales continuarán desprotegidos de las dinámicas transnacionales del mercado que atentan directa y gravemente contra la diversidad cultural y la identidad de los pueblos (Martín Barbero, 2008).

\section{Las industrias culturales y el entorno digital}

El concepto sobre industria cultural surge a finales del siglo XIX, momento en el que las sociedades fueron transformadas por el desarrollo científico y la innovación técnica. A partir de este momento, esta noción sufre una continua evolución en concordancia con el desarrollo de las sociedades y la incorporación de la población a los procesos de producción y consumo. En el año de 1947 los principales teóricos de la Escuela de Frankfurt: Theodor Adorno y Max Horkheimer, definen la industria cultura desde una óptica social y esta adquiere un carácter de cultura de masas. Más tarde, en la década del setenta, las industrias culturales -libro, cine, prensa, música, radio, televisión-, se entienden por su capacidad de producir masivamente contenidos simbólicos y

3 Ver: Fondo Internacional para la Diversidad Cultural (FIDC). Fondo creado como resultado de la Convención de la UNESCO sobre la Protección y la Promoción de la Diversidad de las Expresiones Culturales de 2005, con el fin de promover el desarrollo sostenible y la reducción de la pobreza de los países miembros.

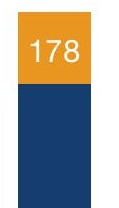


pasan por un proceso de distribución para su consumo. En los años ochenta, el debate se enfocó en torno a la democratización y descentralización cultural, y a partir de la década de los noventa el concepto de industria cultural se vincula directamente con el entorno digital.

En efecto, en tiempos de Internet y de desarrollo de las nuevas tecnologías, las actividades posindustriales y cibernéticas se asocian a diferentes tipos de escenarios y plataformas para el desarrollo de la cadena de valor de las industrias culturales, dando lugar a distintas dimensiones económicas, sociales y procesos de comunicación, así como a otras formas de crear, producir, distribuir y disfrutar de los productos culturales.

El autor Francisco Sierra nos recuerda que:

Los modelos tradicionales de producción y distribución audiovisual en Internet han dado lugar a procesos de migración digital y de multiplicación de los canales de consumo, acceso y visibilidad que, a su vez, originan otros de integración y de capitalización distintos en la cadena de valor, lo que hace factible las posibilidades de proyección de las llamadas cinematografías periféricas apenas exploradas en el nuevo entorno de la Web (pp.13-14).

La cadena de valor en el escenario virtual exige una revisión de conceptos. Así es como la UNESCO (2009), en el Marco de estadísticas culturales, define a la industria cultural -y a la industria creativa-, como: "los sectores de actividad organizada que tienen como objeto principal la producción o la reproducción, la promoción, la difusión y/o la comercialización de bienes, servicios y actividades de contenido cultural, artístico o patrimonial", destacando de esta manera, la creatividad como base de la producción de los bienes, servicios y actividades de contenido cultural, artístico y patrimonial.

En este escenario de expansión de la economía de mercado y desarrollo de los medios masivos de comunicación, tiene lugar también la aceleración de procesos relacionados con la globalización de la cultura y la creciente diversificación de las expresiones culturales. Precisamente es en este marco global en el que las industrias culturales, -dentro del paradigma de la economía política de la comunicación-, se relacionan con las nuevas lógicas de mercado y de consumo.

Precisamente por este motivo es que los procesos de globalización conducen a las industrias culturales a reconfigurar la esfera pública, escenario en el cual la diversidad cultural y los intercambios culturales se posicionan en el centro del debate, apuntalando la discusión sobre de qué manera se garantiza la pluralidad de la creación cultural en un escenario de crisis, convergencia, migraciones, capitalización de lo simbólico. Características propias de las sociedades informatizadas y del conocimiento, en las que, como advierte George Yúdice (2002), realzan el valor de la cultura como recurso.

En tal sentido, al abordar las dinámicas impuestas por los nuevos contextos para la protección y promoción de diversidad de la industria cultural es preciso reflexionar sobre la relación entre diversidad y mercado, y al mismo tiempo, determinar cuáles serían las políticas y estrategias que se deben llevar a cabo con miras a lograr un reconocimiento real de la heterogeneidad y, con ello, orientar nuestras acciones hacia la protección, y promoción de los grupos minoritarios y de sus 
expresiones culturales.

Una de las vías para la protección y promoción de la diversidad cultural la brindan las industrias culturales, y de manera particular, el cine. Esta industria es una herramienta eficaz para la producción y circulación de contenidos simbólicos, debido a su alcance social, y la capacidad de mostrar diversas percepciones del mundo además de transmitir la identidad y valores de los pueblos.

Por su parte, Kulesz (2017) sostiene que "cualquier debate sobre la cultura y la diversidad debe incluir en algún punto la variable de la tecnología digital" (p. 77), debido a que el escenario digital ha cambiado las estructuras del sistema audiovisual en la creación, producción, distribución, exhibición y hábitos de consumo, provocando el interés de organismos internacionales como la UNESCO, así como de las instancias gubernamentales, la sociedad civil, incluida la comunidad académica.

Somos conscientes que la tecnología digital e Internet han cambiado el panorama cultural y nos han dirigido hacia un ecosistema conformado por "nuevos lenguajes, sensibilidades y escrituras por la hegemonía de la experiencia audiovisual sobre la tipográfica, y por la integración de la imagen al campo de la producción del conocimiento, lo cual está incidiendo tanto sobre lo que entendemos por comunicar, como sobre las figuras del convivir y el sentido de lazo social" (Martín Barbero, 2003, s.p.). La diversidad cultural de las historias, la memoria y la expresión cultural de los pueblos, por otra parte, nos brinda la posibilidad de interactuar con estos fenómenos.

De la misma manera, las plataformas digitales ${ }^{4}$ ofrecen a las industrias culturales, entre otras, la ventaja de los nuevos modelos de negocio y financiamiento; maneras alternativas para el funcionamiento de la cadena de valor, nuevas lógicas y actores que están incidiendo en el funcionamiento de las industrias culturales, y que pueden presentar oportunidades de enriquecimiento de la diversidad en términos de producción, difusión, de acceso e intercambio de los contenidos culturales y la ampliación del acceso a un más vasto público.

Siendo ésta una gran ventaja; sin embargo, hay que tener en cuenta que también pueden generar conflictos y desigualdades a la hora de intentar un acceso equitativo de todos los sectores de las sociedades, sobre todo en los países menos desarrollados que pueden contar con menos infraestructura.

La piratería digital, por otra parte, entendida como la infracción deliberada del copyright a escala comercial, es otro problema a tener en cuenta. Esta constituye un fenómeno económico, político y sociocultural que ha generado mucha controversia en cuanto al acceso, circulación y cambios en las formas de consumo de los contenidos culturales. Si bien los contenidos culturales online pueden tener un carácter democratizador, la piratería digital es una fuente de conflictos que afecta la venta legal en su mayor parte de películas, libros, música y, en los últimos años, los videojuegos.

$4 \quad$ Los gigantes de la red; los medios de comunicación por streaming y on demand; los big data y los nuevos modelos de financiamiento en línea, como el crowfounding, etc. 
El entorno virtual supone una mayor posibilidad de circulación, pero también construye modos de percepción homogéneos en la mayoría de los casos. Es necesario tomar en cuenta estos aspectos en la perspectiva de promover la diversidad de las expresiones culturales, ya que, si bien los contenidos manifestados en la industria cultural circulan de una manera que puede favorecer el acceso y la interacción cultural, al no ajustar la diversificación de la oferta queda el riesgo de provocar graves problemas de equilibrio en el intercambio, y como hemos mencionado antes, una fuerte concentración.

\section{Buena práctica para la protección y promoción del cine}

Las dinámicas del mercado actuales y los acuerdos comerciales internacionales, -en las que aparece como una de las protagonistas la industria cinematográfica-, exigen nuevas formas de negociación y medidas de protección para garantizar la diversidad, lograr un equilibrio en el intercambio equitativo y garantizar la heterogeneidad y su acceso.

Como bien sabemos, el desarrollo de la cadena de valor de la industria cinematográfica, en el entorno digital, está condicionado por nuevas formas de poder y por el volumen de mercado. Las grandes majors globalizan la producción cinematográfica aprovechando las nuevas tecnologías y la digitalización, convirtiéndose en estrategias claras para dominar las pantallas a nivel mundial a través de los nuevos formatos televisivos digitales, servicios por cable, on demand, e Internet, proponiendo de esta manera nuevas formas de producción, circulación, exhibición y consumo.

En este plano, es importante mencionar a Retina Latina, uno de los ejemplos paradigmáticos en el campo de las industrias cinematográficas para la protección y promoción del cine en el espacio digital.

Retina Latina es una plataforma online para ver cine latinoamericano. Su lanzamiento fue en el año de 2016, en el marco del Festival de Cine de Cartagena de Indias impulsado por instituciones cinematográficas de Bolivia, Colombia, Ecuador, México, Perú y Uruguay. Esta plataforma se ha convertido en un sistema para responder a las nuevas formas de exhibición y consumo cinematográfico, con esta nueva ventana de Internet, destinada a la exhibición gratuita de contenidos cinematográficos y audiovisuales alternativos, se intenta garantizar un acceso más amplio y democrático a los contenidos cinematográficos de calidad de una manera gratuita a través de Internet. La plataforma es un modelo de cooperación que tiene como objetivo proponer un circuito alterno de circulación y la formación de públicos, promocionando la circulación de las producciones latinoamericanas más allá de sus territorios, así como la creación de redes de producciones latinoamericanas. En el año 2017 Retina Latina fue beneficiaria del premio del Fondo Internacional para la Diversidad Cultural (FIDC) ${ }^{5}$, con el proyecto: Apropiación, fortalecimiento y promoción del cine latinoamericano a través de la plataforma digital regional de cine.

A través de sus objetivos, Retina Latina se propone contribuir a la cooperación Norte-Sur y

$5 \quad$ El Fondo Internacional para la Diversidad Cultural (FIDC) es un fondo creado por mandato de la Convención de 2005 sobre la Protección y la Promoción de la Diversidad de las Expresiones Culturales cuyo fin es promover el desarrollo y la reducción de la pobreza financiando proyectos culturales que protejan y promuevan la diversidad cultural. 
Sur-Sur con estrategias para promover un mejor acceso, circulación y acceso de los bienes y servicios cinematográficos producidos en Latinoamérica a nivel local, regional e internacional, con soluciones digitales e innovadoras tales como: la implementación de aplicaciones para dispositivos móviles; las propuestas de nuevos modelos distribución de las películas y la incorporación de nuevos miembros a la plataforma y la consecuente ampliación del catálogo de las películas disponibles en la plataforma.

\section{Políticas culturales en el entorno digital}

Las dinámicas de las sociedades contemporáneas, la globalización y el desarrollo de las tecnologías de la comunicación, han incidido de una manera determinante en la relación entre la cultura y la política, y por lo tanto, en la formulación de las políticas culturales.

El campo de las políticas culturales ha dejado de estar al servicio exclusivo de los servicios culturales, patrimonio, difusión y el acceso, para sumar otras dinámicas de acción cultural pública basadas en la diversidad, la interculturalidad, los derechos culturales (Nivón, 2013), en donde la estrategia gubernamental exige acciones de mayor eficacia y transversalidad.

La construcción de un proyecto colectivo a futuro, articulado por el Estado, la sociedad civil y diversos actores sociales (Ochoa, 2003) a partir de "profundos replanteamientos de las bases teóricas y de las estrategias de acción tradicional de los Estados en el campo de la cultura, a fin de construir una globalización ascendente" (Jiménez, 2015, p. 37), se apuntan como las directrices dominantes en el direccionamiento hacia un proyecto intercultural acorde con las circunstancias del nuevo momento histórico.

Las industrias culturales se han visto afectadas por los efectos globales, la convergencia de las tecnologías de información y de comunicación: Internet, la televisión digital, los dispositivos móviles. Estos factores conducen a la adopción de otras maneras de gestión y nuevas políticas de protección y de fomento para la defensa de las expresiones culturales.

En este sentido, el estudio de las políticas culturales en la actualidad exige una revisión para adaptar las estrategias a contextos más recientes. Uno de los temas de reflexión más recurrentes en el discurso contemporáneo consiste en determinar ¿cuáles son las políticas públicas que se deben formular e implementar para garantizar la diversidad de las expresiones y contenidos culturales en el entorno digital?, es decir, ¿de qué manera y a través de qué mecanismos deben afrontar los Estados la problemática relacionada con los contenidos culturales digitales?

Si bien esta temática no ha sido afrontada suficientemente por los gobiernos locales, sobre todo en América Latina, si ha concitado el interés de los organismos internacionales, particularmente la UNESCO, al que se han sumado varios sectores de la sociedad civil y a través de la esfera académica ${ }^{6}$. Albornoz y Leiva, (2017a, 2017b), Martín Barbero, (2003), Bustamante, (2011) y Zallo (2017)), como investigadores, entre otros, han abierto un debate sobre la lucha por la diversidad cultural de las industrias culturales en el entorno digital, con el fin de analizar su impacto real en

6 Desde la Universidad Carlos III, el grupo de investigación Diversidad Audiovisual ha impulsado varias propuestas en torno a este tema: http://diversidadaudiovisual.org. 
la sociedad y determinar cuáles son las medidas y estrategias para controlar el desigual flujo de información.

Los cambios provocados por la globalización demandan una revisión del paradigma de las políticas culturales tradicionales y a su vez, la defensa de la diversidad de las industrias culturales implica un "empoderamiento social y diversificado y una puesta al día de herramientas de política cultural y comunicativa para la era digital (Zallo, 2017, p. 99).

En el debate contemporáneo existe un consenso en cuanto al entorno digital y el impacto dual frente a las industrias culturales. Los aspectos positivos de la digitalización e Internet en relación al desarrollo de las industrias culturales pueden constatarse en la posibilidad de ofrecer un mundo sin fronteras, democratizar la información para algunos sectores, y abrir las posibilidades de acceso a los contenidos culturales de cualquier parte del mundo, con el beneficio de una mayor circulación y consumo de contenidos.

Si bien esta constituye una propuesta encomiable, no hay que perder de vista la presencia de amenazas graves a su consecución, particularmente la posible concentración que atenta contra de la diversidad de las expresiones culturales, la inequidad en el acceso, especialmente si pensamos en los sectores tradicionalmente excluidos.

García Canclini \& Martinell nos recuerdan que la lucha por la legitimidad de la democracia y la defensa de la diversidad de la expresiones culturales se ha convertido en uno de los mayores retos en la actualidad, ya que "en la medida en que las industrias audiovisuales y las tecnologías digitales van acercando a las sociedades y engendrando a la vez nuevas diferencias y nuevas desigualdades, ya no principalmente territoriales o de origen histórico, sino según el acceso a los nuevos bienes y mensajes" (2009, p. 12).

La formulación de políticas que tengan como fin la protección y promoción de la diversidad de los bienes y expresiones culturales en la era digital conlleva fomentar una producción diversa y asegurar una distribución justa. Kulesz (2017) mantiene que "la clave estratégica parece radicar en considerar lo digital en términos políticos en lugar de como algo aislado" (p.87). A estas expresiones se unen otras provenientes de organismos internacionales como la UNESCO (2015); así como la de diversos autores como Zallo (2017) y Albornoz y Leiva (2017a), que proponen tomar en cuenta diversos aspectos a la hora de formular las políticas como la diversificación de las fuentes de financiamiento y la aplicación de desgravaciones fiscales para la innovación, el desarrollo de estrategias digitales con componentes culturales y, finalmente, la adaptación de las leyes de creación y propiedad intelectual.

Aunque en la práctica es complejo, se considera necesario formular e implementar políticas culturales dirigidas al fortalecimiento de cada uno de los eslabones de la cadena de valor de las industrias culturales. De acuerdo con Bustamante (2017), la diversidad real de una sociedad depende de la totalidad del proceso de producción, es decir, de su producción y creación, su distribución, comercialización, visibilidad, demanda y consumo efectivo. 


\section{Conclusiones}

Los problemas de la globalización cultural, económica, política y social, la creciente digitalización y sus plataformas han causado importantes cambios en los procesos sociales, por esto la diversidad cultural y sus dinámicas enfrentan un gran reto.

La cultura y sus industrias han adquirido un carácter central para la transformación social, la identidad, la diversidad, la innovación y la creatividad. El papel de las industrias culturales como integradoras sociales y fuentes de desarrollo las han convertido en una herramienta esencial para responder a las dinámicas que se generan en las sociedades actuales.

Al momento de formular una política cultural para la defensa de la diversidad se deben abordar valores de reconocimiento y respeto de lo diferente o minoritario. Se hace necesaria una política que parta de la garantía de los derechos culturales individuales, colectivos y que fomente la cohesión social y las relaciones interculturales. Estas estrategias deben traducirse en políticas públicas y normas que garanticen la diversidad cultural de las industrias culturales en los nuevos entornos.

El impacto del entorno digital en las formas de creación, distribución y consumo cultural, exigen, por un lado, la revisión de las estrategias estatales para la protección y promoción de la diversidad cultural de los pueblos, y por otro, la formulación e implementación de políticas para la creación, distribución y acceso a contenidos culturales.

La Convención de la UNESCO (2005) constituye un ejemplo significativo en el lineamiento de políticas públicas para la defensa de la diversidad y las expresiones culturales. Sin embargo, a pesar de las amenazas que existen entre el entorno digital, las industrias culturales y la diversidad en el proceso de la cadena de valor, no se ha actualizado.

No existen políticas culturales sólidas en las sociedades actuales para la protección y promoción de las industrias culturales y su relación con Internet. En este sentido, es importante aprovechar las oportunidades y anular las amenazas que crea el entorno digital a través de estrategias de gobernanza para el sector cultural y la destinación de recursos para lograr un acceso a las tecnologías más incluyente.

Las políticas culturales para el fomento y la protección de la diversidad en el entorno digital deben estar orientadas, entre otros aspectos, a proteger todos los eslabones la cadena de valor del impacto negativo de los gigantes de la red; a proteger a creadores y usuarios, ampliar las actuaciones, políticas de Estado y programas de cooperación que estimulen la diversidad cultural. Especialmente, una puesta al día en las estrategias de las políticas públicas culturales que correspondan a los nuevos contextos. 


\section{Referencias}

Albornoz, L. y García Leiva, M. (2017a). Diversidad e industria audiovisual. El desafío cultural del siglo XXI. Ciudad de México, México: Fondo de cultura económica.

Albornoz, L. y García Leiva, M. (Eds.) (2017b). El audiovisual en la era digital. Políticas y estrategias para la diversidad. Madrid, España: Cátedra.

Appadurai, A. (2014). Diversidad cultural: una plataforma conceptual. Diversidad cultural, Desarrollo y Cohesión social, 34

Bustamante, E. (2011). Cultura digital: La nueva cultura clásica. Telos. Cuadernos de Innovación y comunicación. La cultura digital, 88, pp. 59-64.

Bustamante, E. (2017). Indicadores y diversidad en la industria audiovisual: la lucha por la diversidad apenas ha comenzado en L. Albornoz, y M. García Leiva (2017). El audiovisual en la era digital. Políticas y estrategias para la diversidad (pp. 137-157). Madrid, España: Cátedra.

García Canclini, N. y Martinell, A. (2009). Diversidad cultural y poder en Iberoamérica. http://www.oei.es/historico/noticias/spip.php?article5250

Jiménez, L. (2015). Políticas culturales y cooperación cultural para la diversidad y la equidad. http://www.oei.es/ historico/pensariberoamerica/ric08a05.htm

Kulesz, O. (2017). La cultura en el entorno digital. https://en.unesco.org/creativity/sites/creativity/files/gmr es.pdf

Martín-Barbero, J. (2003). La globalización en clave cultural: una mirada latinoamericana. Coloquio internacional. https://rei.iteso.mx/bitstream/handle/11117/357/53 02 globalizacion.pdf?sequence=2

Martín-Barbero, J. (2008). Diversidad cultural y convergencia digital. IC Revista Científica de Información y Comunicación, 5, 12-25.

Martinell, A. (2010). Cultura y desarrollo: un compromiso para la libertad y el bienestar. Ediciones AKAL.

Nivón, E. (2013). Las políticas culturales en América Latina en el contexto de la diversidad. En: Hegemonía cultural y políticas de la diferencia (pp. 23-45). Buenos Aires, Argentina: CLACSO.

Ochoa, A. M. (2003) Entre los deseos y los derechos. Un ensayo crítico sobre las políticas culturales. Bogotá, Colombia: ICANH. 
Ochoa, A. M. (2008). Desencuentros entre los medios y las mediaciones: Estado, diversidad y políticas de reconocimiento cultural en Colombia (pp. 153-174). En: M. Lacarrieu y M. Álvarez (Comp.) (2008). La (Ind) Gestión cultural. Una cartografía de los procesos culturales contemporáneos. Buenos Aires, Argentina: La Crujía Ediciones.

Sierra, F. (2013). Diversidad, sociedad de la información y política audiovisual: la experiencia europea. Temas, $74,13-20$.

UNESCO (2005). La Convención para la Promoción y Protección de las Expresiones Culturales. http://unesdoc. unesco.org/images/0014/001429/142919s.pdf

UNESCO (2009). Marco de estadísticas culturales de la UNESCO 2009. http://www.lacult.unesco.org/docc/ Marco estadisticas CLT UNESCO ESP.pdf

UNESCO (2015). Re-pensar las políticas culturales. https://en.unesco.org/creativity/sites/creativity/files/gmr es.pdf

Vich, V. (2014). Desculturizar la cultura. La gestión cultural como forma de acción política.

Yúdice, G. (2017). La diversidad musical en la nube. En: L. Albornoz. y M. García Leiva. (Eds.) (2017b). El audiovisual en la era digital. Políticas y estrategias para la diversidad (pp. 105-132) Madrid, España: Cátedra.

Yúdice, G. (2002). El recurso de la cultura. Barcelona, España: Gedisa Ediciones.

Zallo, R. (2017) Políticas culturales y comunicativas para la diversidad de las expresiones culturales: una aproximación. En L. Albornoz y M. García Leiva. (Eds.) (2017b). El audiovisual en la era digital. Políticas y estrategias para la diversidad (pp.71-103) Madrid, España: Cátedra. 
Copyright (c) 2020 Belén Fiallos Quintero

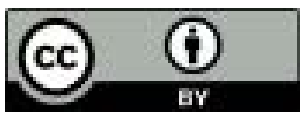

Este texto está protegido bajo una licencia internacional Creative Commons 4.0.

Usted es libre para Compartir-copiar y redistribuir el material en cualquier medio o formato

- y Adaptar el documento - remezclar, transformar y crear a partir del material-para cualquier propósito, incluso para fines comerciales, siempre que cumpla las condiciones de Atribución. Usted debe dar crédito a la obra original de manera adecuada, proporcionar un enlace a la licencia, e indicar si se han realizado cambios. Puede hacerlo en cualquier forma razonable, pero no de forma tal que sugiera que tiene el apoyo del licenciante o lo recibe por el uso que hace de la obra.

\section{Resumen de licencia - Texto completo de la licencia}

